\title{
Base-Region Optimization of SiGe HBTs for High-Frequency Microwave Power Amplification
}

\author{
Zhenqiang Ma, Member, IEEE, and Ningyue Jiang, Student Member, IEEE
}

\begin{abstract}
The base-region optimization of SiGe power heterojunction bipolar transistors (HBTs) for high-frequency microwave power amplification is investigated. It is found that employing a heavily doped base region in conjunction with a high Ge content of proper profile can effectively improve the large-signal power-gain values of SiGe HBTs while maintaining their high breakdown voltages and thus allow them to be efficiently operated with high power at higher microwave frequencies. More importantly, with such a base-region optimization, not only lateral-scaling requirements can be relaxed but also a common-base configuration for power amplification using these devices can be favored, which further enhances the power-gain values of SiGe power HBTs at high frequencies. Load-pull experimental results are presented to show the highest figure-of-merit power performance of SiGe power HBTs with an optimized base region. The power performance of SiGe power HBTs operated at $X$-band with different base-region designs was also compared to illustrate the significant benefits that result from the base-region optimization.
\end{abstract}

Index Terms-Common base (CB), doping profile, figureof-merit (FOM), load pull, microwave, power amplification, power gain, SiGe heterojunction bipolar transistors (HBTs).

\section{INTRODUCTION}

$\mathbf{S}$ IGE HETEROJUNCTION bipolar transistors (HBTs) have undergone substantial development for nearly two decades. Superior RF performance has been demonstrated, through vertical and lateral scalings, on low-power high-speed SiGe HBTs [1]-[3]. Recently, SiGe power HBTs have emerged as competitive alternatives to III-V devices for RF power amplification in wireless handsets and cell phones [4]-[6]. A distinctive feature of these power devices, in comparison to low-power high-speed ones, is their thick and lightly doped collector (epi) layers. With such a collector design, high breakdown voltages have been achieved from these devices to realize high output power and provide sufficient output mismatch ruggedness. Such a collector design has been widely employed in III-V devices [7]. However, due to the lower minority carrier mobility (in the SiGe base region), the lower electron drift velocity, and the smaller bandgap of Si than the III-V counterparts, the achievable Johnson's limit [8] using SiGe power HBTs and their power-handling capability per unit emitter area are not comparable with III-V power devices of similar breakdown voltages. As a result, while present commercial SiGe power

Manuscript received April 5, 2005; revised November 10, 2005. This work was supported by the National Science Foundation under Grant ECS-0323717. The review of this paper was arranged by Editor M. A. Shibib.

The authors are with the Department of Electrical and Computer Engineering, University of Wisconsin-Madison, Madison, WI 53706 USA (e-mail: mazq@engr.wisc.edu).

Digital Object Identifier 10.1109/TED.2006.870279
HBTs can fulfill the RF power level and breakdown-voltage requirements for low-frequency $(0.8-2.4 \mathrm{GHz})$ power amplification [4]-[6], they become incapable to be operated (or with substantially reduced efficiency) at higher RF and microwave frequencies (including 5-6 GHz) when high RF power levels $(>0.5 \mathrm{~W})$ are required. It is obvious that further advancement in device design and optimization is needed in order to make SiGe HBTs (and thus SiGe bipolar BiCMOS) continue to be competitive for future wireless applications of increasing frequencies, in terms of their high-power-amplification capability. As a matter of fact, only limited reports existed in literature on high power $(>0.5 \mathrm{~W})$ amplification at high frequencies $(5 \mathrm{GHz})$ using commercial $\mathrm{SiGe}$ power HBTs [9]. In contrast, III-V power devices (e.g., GaAs HBTs) have demonstrated their capability of high power $(>1 \mathrm{~W})$ amplification with a high power added efficiency (PAE) over a much wider frequency range $(0.9-20 \mathrm{GHz}[10])$ than SiGe HBTs. The straightforward way of further enhancing the power gain of SiGe power HBTs without sacrificing their high breakdown voltages, which are required for power amplification, is further lateral scaling as suggested by Johnson et al. [11]. However, this will inevitably increase the fabrication cost due to the use of smaller lithography feature sizes. In addition, more chip real estate (thus higher cost) may be needed to achieve the same RF power level due to the increased number of electrode contacts (with possible PAE enhancement when employing smaller feature sizes, more RF power output is possible, which can partly compensate the increase of chip area). On the other hand, it is noted that, due to the lack of efficient on-chip powercombining techniques, it is not practical to achieve high power with concurrent high PAE by combining a number of lowpower high-speed SiGe HBTs. Consequently, simple powercombining techniques are not suitable for very hi gh frequency battery-powered wireless communication units requiring a high RF power and a high PAE.

In this paper, we discuss an alternative approach of SiGepower-HBT optimization, the base-region optimization, with the objective of enhancing large-signal power-gain values of these devices at high frequencies. While reporting the highest figure-of-merit (FOM) power performance achieved from $\mathrm{SiGe}$ power HBTs with wide emitter stripes $(2 \mu \mathrm{m})$, fabricated from heterostructures grown by one-step chemical vapor deposition (CVD), we also compare their large-signal power performance between different operation configurations. The advantages of employing the base-region optimization approach will be illustrated by comparing the power performance of SiGe power HBTs with different base-region designs. Finally, we will discuss the practical issues on implementing the optimization 


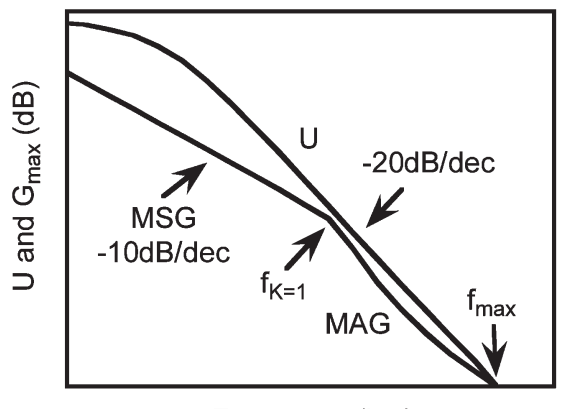

Frequency $(\log )$

Fig. 1. Representative illustration of power gains. Maximum stable/available power gain (MSG/MAG) and unilateral power gain $(U)$ versus the logarithmic frequency for bipolar junction transistors (typical of CE configuration). Since it is MSG and MAG, not $U$, that represent the actual power-gain values and MSG is not directly related to the value of $f_{\max }, f_{\max }$ is not an adequate poweramplification FOM if devices are operated in the frequency range of MSG.

approach in SiGe HBTs that are integrated in commercial SiGe BiCMOS

\section{Considerations of BAse-Region Profile Design}

It has been recognized that the cutoff frequency $f_{T}$, by itself, is not a good FOM for describing the power-amplification capability of semiconductor devices. Instead, the maximum oscillation frequency $f_{\max }$ has been extensively used as a general power-amplification FOM. For bipolar junction transistors, $f_{\text {max }}$ is expressed as

$$
f_{\max }=\left(\frac{f_{T}}{8 \pi R_{B} C_{\mathrm{BC}}}\right)^{\frac{1}{2}}
$$

Since devices are generally operated at frequencies far below $f_{\max }$ in order to have a sufficiently high power-gain value, the operation frequencies fall into the maximum stable gain (MSG) range in most cases (The unilateral power gain $U$ is a deficient FOM in this regard [12]). However, it is noted in Fig. 1 that the MSG values at a certain operation frequency are not directly related to $f_{\max }$ due to the involvement of the stability factor (a detailed justification will become evident in the following text). Obviously, this $f_{\max }$ FOM still has its inherent deficiency if it is used as an indicator of the power-amplification capability when devices are operated in the MSG frequency range. Therefore, it is impervious to examine the actual power-gain values at specific operation frequencies when considering the poweramplification capability of devices.

In our recent paper [12], it has been shown that the MSG for the common-emitter (CE) configuration is

$$
\mathrm{MSG}_{\mathrm{CE}}=\frac{\alpha}{\omega R_{E} C_{\mathrm{BC}}}
$$

where $C_{\mathrm{BC}}$ is the base-collector $(\mathrm{B}-\mathrm{C})$ junction capacitance and $R_{E}$ is total emitter resistance, including parasitic emitter resistance $R_{\mathrm{ex}}$ and intrinsic emitter resistance $R_{e}\left(=\alpha / \mathrm{g}_{m}=\right.$ $\alpha k T / I_{C}, k T=26 \mathrm{meV}$ ), respectively. From (2), it is evident that the optimization of the base region (e.g., reducing the base resistance $R_{B}$ ) will not affect power-gain values [but will affect the $f_{\max }$ value as seen from (1)] when devices are operated in the MSG frequency range under the CE configuration. (It is noted that although the reduction of $R_{\mathrm{ex}}$ can improve $\mathrm{MSG}_{\mathrm{CE}}$, there is not much additional room under this option for modern SiGe HBTs.) However, if the base region is optimized such that base resistance $R_{B}$ can be reduced, the maximum available power gain (MAG) can be improved as can be seen from

$$
\mathrm{MAG}=\operatorname{MSG}\left(K-\sqrt{K^{2}-1}\right)
$$

where $K$ is the Rollett's stability factor [13]; for the CE configuration, it can be expressed as [12]

$$
K_{\mathrm{CE}} \approx\left[\left(R_{\mathrm{ex}}+2 R_{B}\right) C_{\mathrm{BC}}+R_{e} C_{\pi}\left(1+\frac{2 R_{B}}{R_{\mathrm{ex}}}\right)\right] \omega
$$

Thus, it can be concluded that, for the CE configuration, the base-region optimization for the purpose of reducing the base resistance only affects power-gain values of SiGe HBTs when they are operated in the unconditionally stable (upper MAG) high-frequency range. In terms of the fact that $f_{\max }$ does not correlate with the values of MSG, the above analysis also proves that $f_{\max }$ is a deficient power-amplification FOM when devices are operated in the MSG frequency range under the $\mathrm{CE}$ configuration. For most semiconductor devices, power-gain values (MAG) in the high-frequency range are much smaller than that of the MSG (Fig. 1). For this reason, most devices are operated in the MSG range instead of the MAG frequency range.

Contrary to the case of the $\mathrm{CE}$ configuration, for the common-base (CB) configuration, the base-region optimization certainly improves power-gain values (MSG) as shown in the following [12]:

$$
\mathrm{MSG}_{\mathrm{CB}}=\frac{\alpha}{\omega R_{B} C_{\mathrm{BC}}} .
$$

Again, it is noted that the $f_{\max }$ shown in (1) does not completely depict the power-amplification capability of devices when they are operated in the MSG frequency range under the $\mathrm{CB}$ configuration, because $f_{\max }$ (and thus the extrapolated power-gain values based on the $f_{\max }$ value) is related to $R_{B} C_{\mathrm{BC}}$ (1) (with $f_{T}$ entanglement) in a different fashion from that of the $\mathrm{MSG}_{\mathrm{CB}}$ in relation to $R_{B} C_{\mathrm{BC}}$ (5). Similar to the case of the CE configuration, the MAG can also be improved with the base-region optimization in the $\mathrm{CB}$ configuration, as it is clear from (3) and (6) that [12]

$$
K_{\mathrm{CB}} \approx\left[\left(R_{B}+2 R_{\mathrm{ex}}\right) C_{\mathrm{BC}}+R_{e} C_{\pi}\right] \omega
$$

From the above analyses, one can see that without changing the device's emitter and collector parameters, $\mathrm{MAG}_{\mathrm{CE}}, \mathrm{MSG}_{\mathrm{CB}}$, and $\mathrm{MAG}_{\mathrm{CB}}$ can be readily improved with the reduction of only the base resistance. Without substantial lateral scaling of the emitter-finger width, the most effective approach to reduce the base resistance is to increase the doping concentration of the intrinsic base region while maintaining a low-resistance extrinsic base region [1]. For mesa-type SiGe power HBTs 
[14]-[16], the total base resistance can be simply improved by only increasing the base doping concentration of the SiGe base region. In comparison to the alternative approach of improving $f_{\max }$ via improving $f_{T}$ (1) (through vertical scaling) to improve power-gain values, for which the breakdown voltage is consequently subject to the reduction resulting from the restriction of Johnson's limit [8], the current approach of base-region optimization needs not to sacrifice the breakdown voltages (no change of collector parameters is required) of SiGe HBTs. Therefore, the mismatch ruggedness and the larger voltage swing can still be maintained for power-amplifier applications using this approach.

As the base doping concentration is increased, the current gain $\beta$ of CE HBTs (not important for CB HBT, as $\alpha$ is less than unity) will be inevitably reduced. To restore the current gain $\beta$, one can increase the Ge content in the base (within the Ge strain limit), which gives rise to a larger band offset between $\mathrm{Si}$ (emitter) and $\mathrm{SiGe}$ (base) [17]. However, if the base doping concentration is extremely high, a significantly enhanced recombination will be induced in the base region. Due to the enhanced base-region recombination, the additional current gain restored with the "extra" Ge content in the base can be partially or completely compromised (detailed discussions of the current-gain dependence of CE SiGe HBTs on the Ge content/profile and the base-region recombination will be reported later). In this case, a further increase of the Ge content in the base region will no longer be effective in helping restore the current gain. As a result, if CE HBTs are to be used for power amplification (note that the optimization approach works for CE HBTs only if the devices are operated in the MAG frequency range) and a high current-gain value is also simultaneously needed, the base-region optimization approach described herein should not be considered. In this paper, we mainly demonstrate the effectiveness of the base-region optimization approach for CB SiGe HBTs.

Due to the degradation of the electron mobility in the base region resulting from employing a heavy doping level, the base transit time $\left(\tau_{B}\right)$ will be degraded. While the total emitterto-collector transit time $\left(\tau_{\mathrm{EC}}\right)$, thus $f_{T}$ (only for CE HBTs), will be degraded (the degradation of $f_{T}$ will not be significant because $\tau_{B}$ is not a dominant component in $\tau_{\mathrm{EC}}$ for highbreakdown-voltage SiGe HBTs [18]), the power-gain values shown in (2)-(6) are not to be degraded.

It is important to notice that a significant advantage of using a heavy base doping concentration associated with a high $\mathrm{Ge}$ content is the relaxation of the emitter current crowding effect. With significantly reduced intrinsic base sheet resistance, the emitter-finger width can thus be made wider than the case where a lightly doped base region is employed. Owing to the feasibility of using wider emitter-finger stripes, a higher power density and a more economic use of the chip real estate for SiGe power HBTs can thus be realized using the base-region optimization approach described above.

\section{Design And Fabrication of SiGe Power HBTs}

Based on the above considerations, the design goal for power SiGe HBTs is to achieve high power-gain values at high oper- ation frequencies, while maintaining high breakdown voltages and suppressing adverse thermal effects. For the heterostructure design of SiGe HBTs, the doping concentration and the thickness of each layer need to be carefully selected. A detailed discussion on the considerations of selecting these parameters can be found in our previous paper [14]. In comparison to the previous SiGe-power-HBT structure [14], only the base region is redesigned for this heterostructure, and other parameters have been kept identical. Since the collector layer is kept the same (500-nm thickness and $3-4 \times 10^{16} \mathrm{~cm}^{-3}$ doping concentration), the high-breakdown-voltage characteristics of HBTs made from this heterostructure are expected to be maintained. Nonetheless, in this SiGe HBT heterostructure, the peak base doping concentration has been increased by over three times (a factor of 3.25) from the previous $2.4 \times 10^{19} \mathrm{~cm}^{-3}$ to the current $7.8 \times 10^{19} \mathrm{~cm}^{-3}$. A box-type Ge profile is still used for this heterostructure. However, the Ge content is increased from the previous $21 \%$ [14] to $25 \%$, and the Ge width is reduced from the previous $32 \mathrm{~nm}$ to $22 \mathrm{~nm}$ for this structure. Considering the major change of base doping concentration and the enhanced recombination resulting from the doping increase in the base, the changes on the Ge content and width are not expected to exhibit a dominant role on the overall change of the base resistance or on the overall change of the current gain for CE HBTs. In accordance with the reduction of Ge width, the base doping profile width (characterized with full-width at half-maximum (FWHM)) of the current SiGe heterostructure is also reduced. Due to the reduction of the base doping profile width, the increase factor of the total base doping concentration from the previous one will be less than 3.25. The measured secondaryion mass-spectroscopy (SIMS) profile of the new SiGe HBT heterostructure (base-"optimized," ibid. The quotation mark implies that further optimization may still be possible) is shown in Fig. 2(a). For comparison, the SIMS profile of the previous HBT heterostructure (replotted) [14] (denoted as not-optimized in the relevant figures) is shown in Fig. 2(b). To characterize the base sheet resistance, TLM patterns were prepared on exposed (using selective $\mathrm{KOH}$ etching) SiGe base layer. The measured base sheet resistance of the base-"optimized" SiGe HBT heterostructure and the previous one [14] are $1160 \Omega / \square$ and $2960 \Omega / \square$, respectively. The improvement on the base sheet resistance for the new heterostructure is mainly ascribed to the increase of the base doping level and, as the secondary effect, to the enhanced hole mobility due to the increased Ge amount.

For the lateral layout design of the SiGe HBT, all dimensions are kept identical to the previous one [14]. The distributed subcell structure is used with two fingers in one subcell and with $2 \mu \mathrm{m}$ for each emitter finger. The total emitter areas of the 10-emitter-finger and the 20-emitter-finger SiGe HBTs are 600 and $1200 \mu \mathrm{m}^{2}$, respectively. For further details of the layout structure, please refer to our previous paper [14]. The heterostructure was grown using one-step CVD on 4-in high-resistivity $\mathrm{Si}$ wafers. The heterostructure was then fabricated into mesa-type multifinger devices using a double-mesa process, of which the fabrication details can be found in a publication by Rieh et al. [15]. It is noted that a "cold" process was used to fabricate the mesa-type HBTs in both the previous 


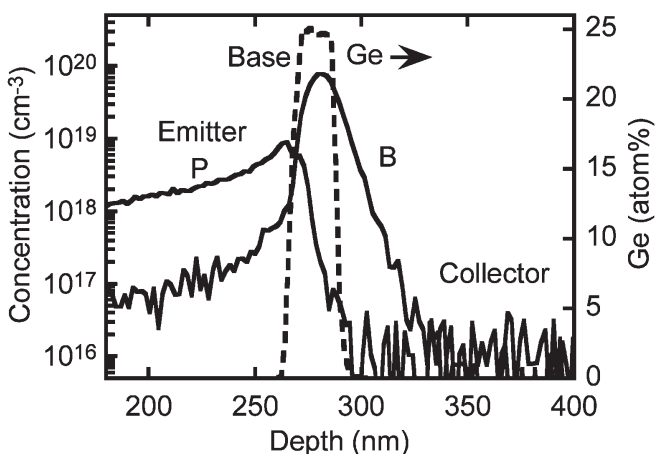

(a)

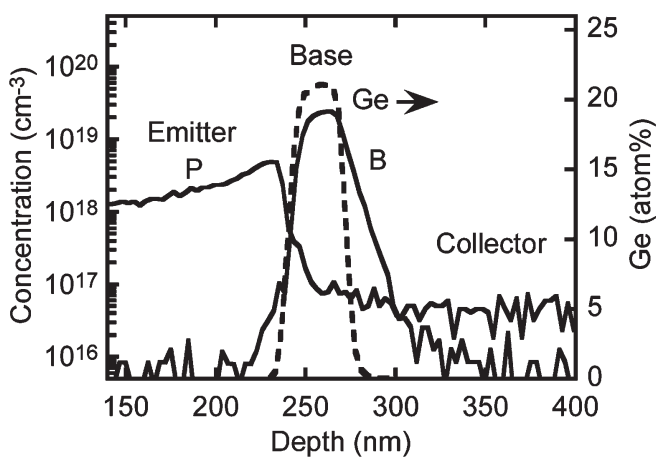

(b)

Fig. 2. (a) SIMS profile of base-"optimized" SiGe HBT heterostructure with a peak base doping concentration of $7.8 \times 10^{19} \mathrm{~cm}^{-3}$ and a box-type Ge profile of 22-nm width. (b) SIMS profile of previous SiGe HBT heterostructure with a peak base doping concentration of $2.4 \times 10^{19} \mathrm{~cm}^{-3}$ and a box-type Ge profile of 32-nm width for comparison [14].

[14] and the current papers. In comparison to the "hot" process used for commercial SiGe HBTs, a reduced current gain at low bias is generally observed due to the poor surface passivation from the "cold" process. Such "cold"-process-related-device electrical characteristics have been described previously [14], [15]. Since high bias levels are used for the power amplification, no adverse effects are expected from using the "cold" process for device fabrication.

\section{DC AND SMALl-SignAl CharaCtERISTICS}

The dc characteristics of the base-"optimized" SiGe HBTs were measured and compared with the previous SiGe HBTs [14] of identical size. The following discussion will focus on the characteristics of ten-emitter-finger device for an easy comparison. The measured dc current gain as a function of collector current of a base-"optimized" CE SiGe HBT is shown in Fig. 3 with the current gain measured from a previous CE SiGe HBT (not-optimized) [14] for comparison. It can be seen that both devices show low current-gain values, which are due to the use of low emitter doping concentrations and high base doping concentrations in the devices. Furthermore, lower current-gain values were measured from the base-"optimized" device than those obtained from the previous one. This is ascribed to the higher base doping level used in the base-"optimized" device (emitter doping profiles are the same for both heterostructures as seen in Fig. 2). The measured breakdown-voltage value

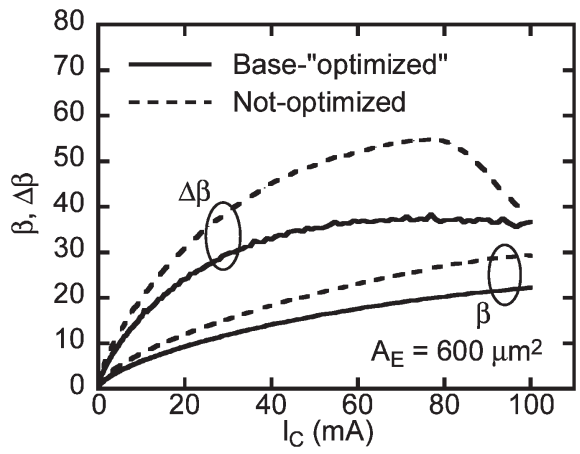

Fig. 3. Measured current gain $\beta$ and incremental current gain $\Delta \beta$ as a function of the collector current for a base-"optimized" CE SiGe HBT and the previous CE SiGe HBT. The bias voltages used for these two devices are $V_{\mathrm{CE}}=7.5 \mathrm{~V}$ and $7.0 \mathrm{~V}$, respectively. The maximum collector current (100 mA) is limited by Agilent $4155 \mathrm{~B}$ semiconductor parameter analyzer.

$\mathrm{BV}_{\mathrm{CBO}}$ of the base-“optimized" SiGe HBT is about $24 \mathrm{~V}$, which is very close to that of the previous one. However, the measured $\mathrm{BV}_{\mathrm{CEO}}$ value from the base-"optimized" devices is $9.5 \mathrm{~V}$, which is slightly higher than the previous value $(8.1 \mathrm{~V})$. The higher $\mathrm{BV}_{\mathrm{CEO}}$ obtained from the base-"optimized" devices (besides the difference of $\mathrm{BV}_{\mathrm{CBO}}$ ) is mainly caused by lowered current-gain values of these devices as dictated by

$$
\mathrm{BV}_{\mathrm{CEO}}=\frac{\mathrm{BV}_{\mathrm{CBO}}}{\beta^{\frac{1}{n}}} .
$$

The improved $\mathrm{BV}_{\mathrm{CEO}}$ thus allows a higher bias voltage $\mathrm{V}_{\mathrm{CE}}$ to be applied to the devices for the current-gain measurement as shown in Fig. 3. In Fig. 3, the incremental current gain $\left(\delta \beta=\Delta I_{C} / \Delta I_{B}\right)$ is also measured from these devices as a function of the collector current. It is found that lower incremental current-gain values were measured from the base"optimized" device than the previous one. Since the incremental gain is a rough approximation of the differential current gain $\left(d I_{C} / d I_{B}\right)$, the lowered incremental current gain also indicates a smaller $f_{T}$ (ac gain) of the base-"optimized" device than the previous one.

Fig. 4 shows the small-signal characteristics (without deembedding) measured from the base-"optimized" SiGe HBT. For comparison, the small-signal characteristics measured from the previous SiGe HBT were also plotted. Both devices were biased at the same optimum bias values (to achieve peak $f_{T}$ ) with $V_{\mathrm{BE}}=1.13 \mathrm{~V}$ and $I_{C}=66 \mathrm{~mA}$. In Fig. 4(a), both the ac current gain and the power gain versus frequency under the $\mathrm{CE}$ configuration are plotted. A lower current gain and a lower $f_{T}$ than the previous one were measured from the base"optimized" SiGe HBT, owing to the degradation of $\tau_{B}$ as described in Section II. Nevertheless, improved MAG values were measured from the base-"optimized" device, indicating the improvement of the base resistance (2), (3). Since the emitter resistance was not changed (same emitter and emitter contact layers yield the same $R_{\mathrm{ex}}$ and same bias current yields the same $R_{e}$ ), not much difference was observed on the values of the MSG between the two devices. The frequency point at which stability factor $K=1$ shows a right shift (to higher frequency), 


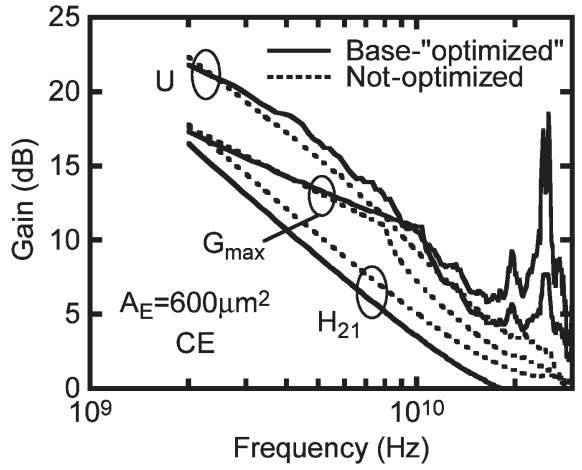

(a)

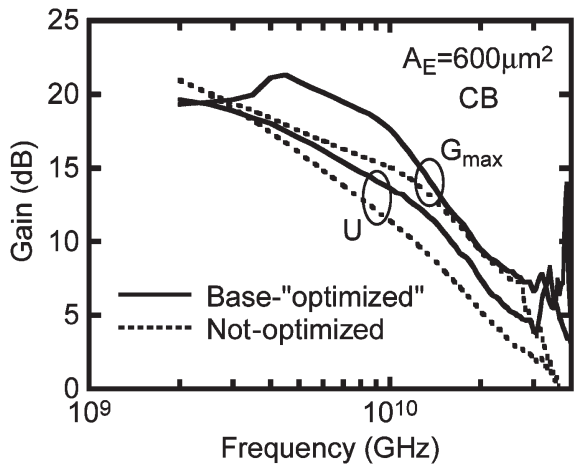

(b)

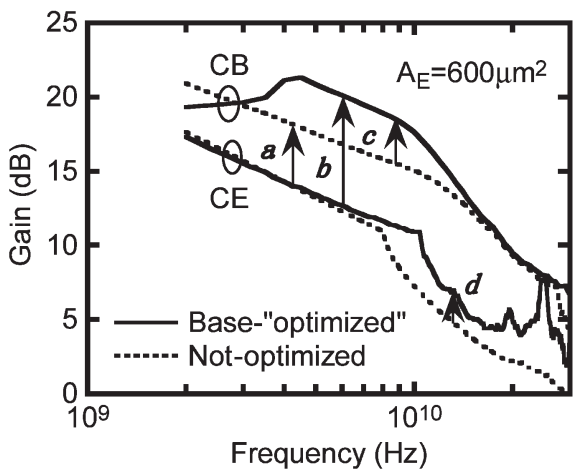

(c)

Fig. 4. Measured small-signal characteristics for the base-"optimized" SiGe HBT (solid lines) and the previous SiGe HBT (dashed lines). The two devices have the same layout structure, and both have ten-emitter fingers with a total emitter area of $600 \mu \mathrm{m}^{2}$. (a) Devices are operated in the CE configuration and with optimum bias: $V_{\mathrm{BE}}=1.13 \mathrm{~V}$ and $I_{C}=66 \mathrm{~mA}$. (b) Devices are operated in the $\mathrm{CB}$ configuration with bias: $V_{\mathrm{EB}}=-1.33 \mathrm{~V}$ and $I_{C}=77 \mathrm{~mA}$. (c) Comparison of small-signal power-gain $\left(G_{\max }\right)$ characteristics between $\mathrm{CE}$ and $\mathrm{CB}$ configurations for both the base-"optimized" and the previous SiGe HBTs.

which is also due to the improvement of the base resistance (3). Based on Fig. 4(a), it can be seen that the improvement of the base resistance extends the frequency range of the MSG and enhances the values of MAG. The measurement results are consistent with the analysis made in Section II.

Fig. 4(b) compares the power-gain characteristics of the two SiGe HBTs under the CB configuration. It is observed that, due to the base-resistance improvement, the MSG is significantly improved. Specifically, the small-signal powergain value $\left(G_{\max }\right)$ at $8.4 \mathrm{GHz}$ (to be used for load-pull characterizations) was increased from 15.8 to $18.8 \mathrm{~dB}$ and more power-gain improvement is obtained in the frequency range below $8.4 \mathrm{GHz}$. The improvement of the MSG results from the base-region optimization as indicated in (5). A slight improvement of the MAG is also observed as shown in Fig. 4(b), which is also consistent with (3) and (6). Simulation studies using MEDICI also prove that the improvement of MAG is not so effective as that of the MSG with the increase of the base doping concentration under a CB configuration. In Fig. 4(c), the small-signal characteristics between $\mathrm{CE}$ and $\mathrm{CB}$ configurations are compared for both the base-"optimized" and the previous SiGe HBTs. It is evident that the CB configuration provides higher MSG and MAG values than that of the CE configuration for both the base-"optimized" device and the previous one in the characterized frequency range. This is due to the fact that similar doping profiles (i.e., the base doping level is higher than the emitter doping level) are used in both devices; for such type of doping profiles, the MSG and the MAG of the $\mathrm{CB}$ configuration are higher than the $\mathrm{CE}$ configuration. For details, please refer to our recent paper [12]. More importantly, with a further increase of the base doping concentration in the base-"optimized" device, the improvement of MSG values resulting from using the $\mathrm{CB}$ configuration is further dramatically enhanced (by comparing arrows $a$ and $b$ in Fig. 4(c)). The enhancement of the MSG resulting from the increase of the base doping level under the $\mathrm{CB}$ configuration is more than that of the MAG under the CE configuration (by comparing arrows $c$ and $d$ ). From the measurement results shown in Fig. 4(c), it is clear that the base-region optimization is effective to improve $\mathrm{MSG}_{\mathrm{CB}}$ and $\mathrm{MAG}_{\mathrm{CB}}$. Moreover, $\mathrm{MSG}_{\mathrm{CE}}$ is extended into higher frequencies. Since most power devices are operated in the MSG frequency range, the power-gain benefits obtained from using the base-region optimization and, particularly, from employing the $\mathrm{CB}$ configuration are thus very significant. Additionally, from these measurement results (Fig. 4), it is also unambiguous that $f_{\max }$ itself is not a perfect FOM to indicate the power-amplification capability of devices unless the operation frequencies are specified.

\section{LARGe-Signal Performance Characteristics}

In order to compare the large-signal power-performance characteristics between two SiGe HBTs, load-pull measurements were performed on the base-"optimized" SiGe HBT. For a direct comparison with the previous design, an operation frequency of $8.4 \mathrm{GHz}$, which is the same as that of the previously reported [14], was used for source/load-pull characterizations. Fig. 5(a) shows the comparison of power-performance characteristics between these two HBTs (both have ten emitter fingers, total $A_{E}=600 \mu \mathrm{m}^{2}$ ) under the $\mathrm{CB}$ configuration. The base"optimized" device was also biased in the class AB mode using comparable bias values with the previous one [14] and was also optimized for maximum $P_{\text {out }}$. Evidently, an improved power performance was measured from the base-"optimized" SiGe HBT under the same bias as the previous one, with the enhanced output RF power $P_{\text {out }}$, power gain $G$, and PAE. The improved power gain (also seen from Fig. 4(b) for small-signal powergain values) in the optimized device permits more dc power to be converted to RF power. As a result, a higher RF output 


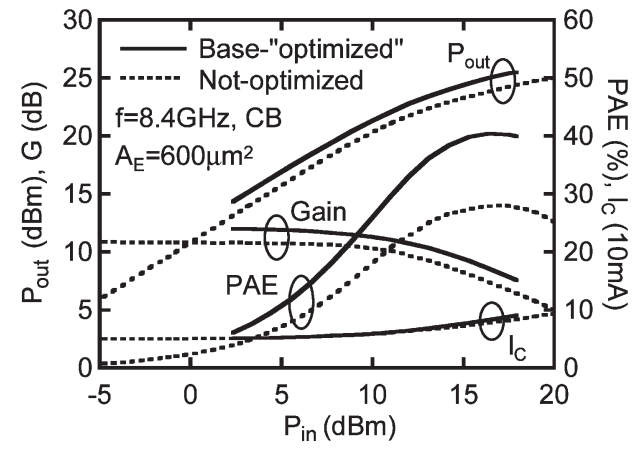

(a)

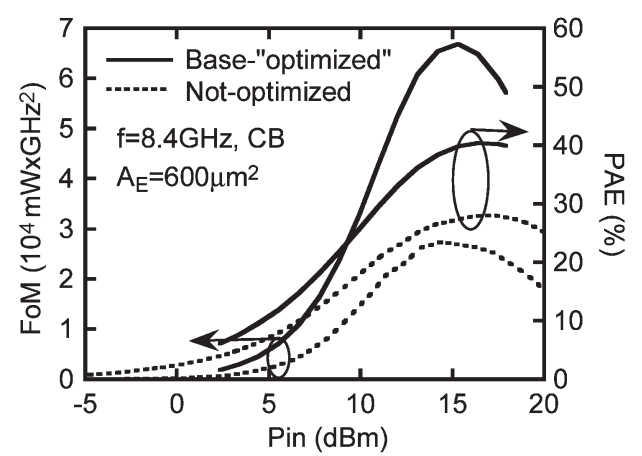

(b)

Fig. 5. (a) Measured large-signal characteristics for the base-"optimized" SiGe HBT (solid lines) and the previous SiGe HBT (dashed lines) with the same layout structure and the same total emitter area of $600 \mu \mathrm{m}^{2}$. Both devices are operated in the CB configuration with identical bias, which can be seen from the measured collector current. (b) Calculated power-performance FOM as a function $P_{\text {in }}$ based on the measurement results shown in (a). The PAE versus $P_{\text {in }}$ was plotted again for reference.

power is measured. The improved $P_{\text {out }}$ further results in an improved PAE $\left(\mathrm{PAE}=\left(P_{\text {out }}-P_{\mathrm{in}}\right) / P_{\mathrm{dc}}\right)$. In order to quantify the improvement of the power performance, the poweramplifier FOM is borrowed from the International Roadmap for Semiconductors (ITRS) [19] to convert the power-performance parameters into one comparable value. This FOM is defined as

$$
\mathrm{FOM}=P_{\mathrm{out}} \cdot G \cdot \mathrm{PAE} \cdot f^{2}\left(\mathrm{~mW} \cdot \mathrm{GHz}^{2}\right) .
$$

Using this FOM, the power-performance comparison results are plotted in Fig. 5(b). It is noted that the highest FOM occurs near (earlier than) the peak PAE point. Moreover, it is found that a 2.5-times improvement in the FOM has been achieved from the base-"optimized" device. The power-performance results of the previous SiGe HBT under CE configuration was not available due to the fact that the power gain at $8.4 \mathrm{GHz}$ is too low (only 9.4-dB MAG, as seen in Fig. 4(a)). In other words, the power-amplification frequency has been extended using the CB configuration and further extended via the baseregion optimization (Fig. 4(c)).

Fig. 6 shows the power-performance characteristics of a 20emitter-finger base-"optimized" SiGe HBT under both CE and $\mathrm{CB}$ configurations. Due to much lowered power-gain values of previous SiGe HBTs with 20-emitter fingers at $8.4 \mathrm{GHz}$, the large-signal power performance was not measured from those devices, indicating their incapability of power amplifica-

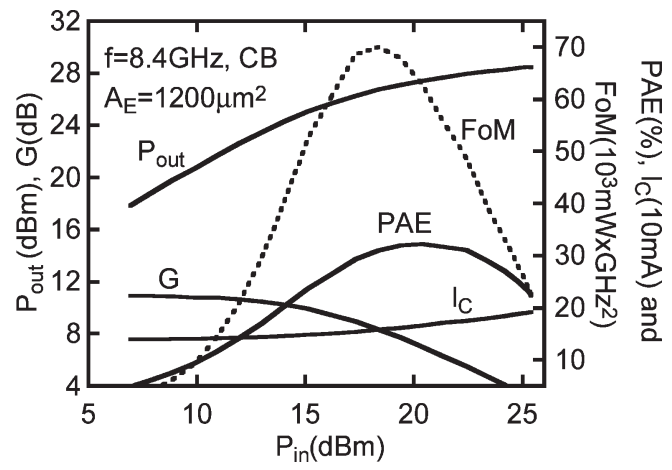

Fig. 6. Measured large-signal characteristics for base-"optimized" SiGe HBT (solid lines) with a total emitter area of $1200 \mu \mathrm{m}^{2}$. The calculated power performance in terms of the FOM based on the measurement results was also plotted (dashed lines).

tion at $8.4 \mathrm{GHz}$ irrespective of $\mathrm{CE}$ or $\mathrm{CB}$ configurations. By comparing the measured power-performance results between $\mathrm{CE}$ and $\mathrm{CB}$ configurations for the 10-emitter-finger (Fig. 7(a)) and the 20-emitter-finger SiGe HBTs (Fig. 7(b)) where both devices are base-"optimized", one can see that a much higher power performance was achieved from using the $\mathrm{CB}$ configuration than the $\mathrm{CE}$ configuration. At the same $P_{\mathrm{in}}$, higher power-gain values and higher $P_{\text {out }}$ levels were obtained at $8.4 \mathrm{GHz}$. This is consistent with the measured small-signal power-gain values (Fig. 4(c)). In Fig. 7(b), the FOM (8) measured for the 20-emitter-finger CB SiGe HBT was also plotted. At $P_{\text {in }}=18.4 \mathrm{dBm}$, an FOM value equal to $7 \times$ $10^{4} \mathrm{~mW} \cdot \mathrm{GHz}^{2}$ has been achieved. To our knowledge, this is the highest power-performance FOM that is ever achieved from any single SiGe HBT operated at any frequency. However, it is noted that this state-of-the-art FOM was achieved from an SiGe HBT with a large width of emitter-finger stripes $(2 \mu \mathrm{m})$, indicating the effectiveness of the base-region optimization on the improvement of the power performance for SiGe HBTs. It is speculated that even higher FOM values can be achieved and higher operation frequencies can be extended into with proper downscaling of the emitter-finger stripes. For comparison, the recently reported best power-performance FOM values $\left(6.3-6.9 \times 10^{4} \mathrm{~mW} \cdot \mathrm{GHz}^{2}\right)$ from commercial SiGe HBTs are achieved using much smaller (0.8 [20] and $0.9 \mu \mathrm{m} \mathrm{[4])} \mathrm{emitter-}$ finger stripes. Furthermore, although comparable FOM values with the ones developed in this paper have been demonstrated from these commercial devices, these commercial devices are only suitable for low-frequency $(0.8-6 \mathrm{GHz})$ power amplifications and are incapable of power amplification at $8.4 \mathrm{GHz}$ or higher. On the other hand, although the highest FOM has been achieved from 20-emitter-finger SiGe HBTs, the ratio (7/6.69) between this FOM and that of the ten-emitter-finger device (Fig. 5(b)) is not proportional to the ratio (2/1) of the areas of the two devices, indicating that further optimizations on other effects (e.g., thermal effects and parasitics) are required as the area of power devices becomes larger.

To summarize, the highest FOM achieved from our SiGe power HBTs results from the following device-optimization and operation-configuration aspects: the base-region optimization for a low base resistance to achieve adequate power-gain values at a high operation frequency $(8.4 \mathrm{GHz})$; employment 


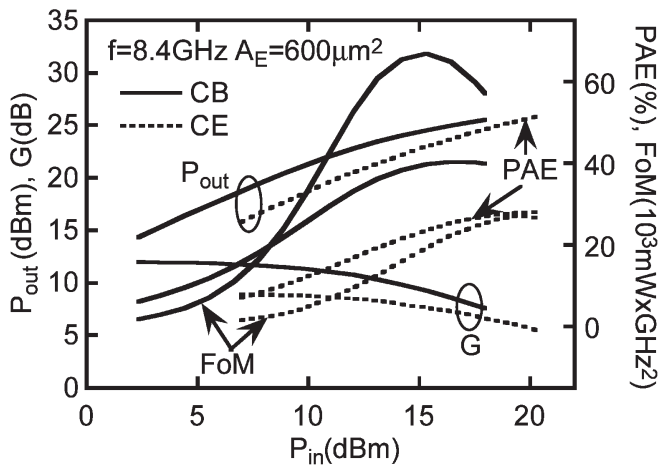

(a)

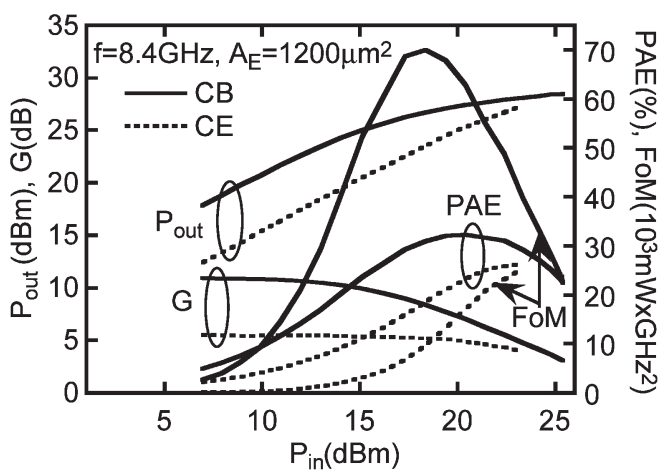

(b)

Fig. 7. (a) Comparison of the measured large-signal power performance and the calculated FOM between CE and CB configurations for base-"optimized" 10-emitter-finger SiGe HBTs $\left(\mathrm{A}_{E}=600 \mu \mathrm{m}^{2}\right)$ and (b) 20-emitter-finger SiGe HBTs $\left(\mathrm{A}_{E}=1200 \mu \mathrm{m}^{2}\right)$.

of a $\mathrm{CB}$ configuration to extract higher power-gain values than available from a $\mathrm{CE}$ configuration; high operation voltage resulting from using a thick and lightly doped collector to allow a high-power operation; and minimized thermal effects [14].

\section{FURTHER DiscusSiONS}

In this section, some practical issues of employing the baseregion optimization approach are discussed.

Power SiGe HBTs in Commercial SiGe BiCMOS: Maintaining a high breakdown voltage for SiGe HBTs allows a high-voltage swing amplitude and thus a high power level to be delivered by these devices. In addition, an adequate mismatch ruggedness, which is essential for the reliability of power-amplifier applications, can be maintained with high breakdown voltages. Due to the need of a thick and lightly doped collector layer to realize high breakdown voltages, it is impossible to achieve a high $f_{T}$ value from high-breakdownvoltage SiGe HBTs. Apparently, achieving high power-gain values through optimizing the $f_{T}$ is not viable. Instead, the optimizations of $R_{B}$ and $C_{\mathrm{BC}}$ become very effective to enhance the power-gain values of $\mathrm{SiGe}$ power HBTs. With the shallow trench or oxide isolation in commercial SiGe HBTs, the room for a substantial reduction of $C_{\mathrm{BC}}$ becomes rather limited. Instead, more room for the reduction of the base resistance may still exist. While the intrinsic base resistance of SiGe HBTs can be straightforwardly reduced by increasing the base doping level, the reduction of the extrinsic base resistance will require more advanced design and processing techniques [1]. Within the current BiCMOS-technology scheme, the same SiGe epilayer is commonly shared among all types (e.g., different breakdown voltages) of SiGe HBTs. For some of these devices, high current-gain values are needed for certain circuit applications. Since using the base-region optimization approach described herein will result in reduced $\beta$ values for all CE HBTs and the entire BiCMOS platform has to meet the requirements of a variety of circuit applications, the base-region optimization approach described herein thus becomes difficult to directly adopt. An alternative option to adopt the optimization approach is to customize the commercial SiGe HBTs for high-power amplification by optimizing the base regions of these devices and integrate these base"optimized" SiGe power HBTs with CMOS. Another option to integrate the base-"optimized" SiGe power HBTs into the existing SiGe BiCMOS platform, which may be considered as a future $\mathrm{SiGe} \mathrm{BiCMOS}$, is to separately optimize the base regions of the high-breakdown-voltage SiGe power HBTs (e.g., grow a separate $\mathrm{SiGe}$ epilayer with a high doping concentration to achieve a low base resistance). With regard to the suppression of boron outdiffusion in light of the heavy doping level used for the base-region optimization, introducing carbon into the $\mathrm{SiGe}$ epilayer has been proven to be effective [21], [22]. To date, this carbon doping technique has been used in many commercial SiGe HBTs [23], [24].

Configurations: Traditionally, a CE configuration for bipolar junction transistors (including SiGe HBTs) has been employed in most circuit applications. However, our recent study [12] has shown that, in terms of power-gain values, there is a vast "market" of using a CB configuration for power amplification, particularly, at high frequencies (relative to the $f_{\max }$ value) of devices. As the total base resistance is reduced, we expect a much bigger "market" of using a CB configuration for power amplification due to the fact that higher power-gain values in a $\mathrm{CB}$ configuration than in a $\mathrm{CE}$ configuration are available in a much wider frequency range (relative to the $f_{\max }$ value) [12] see (Fig. 4(c)). While using a CB configuration for power amplification in $\mathrm{SiGe}$ BiCMOS does not require any further processing advancement, a higher breakdown voltage $\left(\mathrm{BV}_{\mathrm{CBO}} \gg \mathrm{BV}_{\mathrm{CEO}}\right)$ and thus higher output mismatch ruggedness are available, which is a significant benefit obtained at no extra cost. Moreover, with the "extra" breakdown-voltage margin in $\mathrm{CB}$ configuration, the thickness of the collector layer of SiGe HBTs can be reduced. Correspondingly, a higher collector doping level can be allowed in the collector layer for a higher RF power density.

Cost Issues: There has been an everlasting debate on the cost of $\mathrm{SiGe}$ BiCMOS in comparison to III-V device technologies (e.g., InGaP/GaAs) for power amplification in the frequency range where both types of devices are capable of performing the same functions [25]. One of the major supports for the claim that SiGe BiCMOS is not less expensive than the III-V counterpart is that more chip area will be consumed by SiGe HBTs in order to achieve the same RF power level with comparable PAE (other supports include the processing complexity of $\mathrm{SiGe}$ BiCMOS). The underlying reason for demanding more chip area by SiGe HBTs is that the power density available from $\mathrm{Si}$ 
(as a collector) is smaller than GaAs, which is directly related to bandgap difference between these two materials. Besides the material properties, the downscaling of SiGe HBTs (in order to achieve a higher operation frequency or a higher power gain at certain operation frequencies) requires that more electrodecontact area be used for the fine-shaped multifingers in highpower devices. Such fine-fingered device structures occupy an expanded chip real estate in comparison to III-V power devices for which wider fingers are currently used. With the base-region optimization strategy demonstrated in this study, the lateral scaling of SiGe HBTs can be dramatically relaxed without modifying the extrinsic base region. As a result, wider emitter stripes (with only a moderate level of lateral scaling) can be used to increase the average power density of SiGe power HBTs and consequently to reduce the required chip area for a certain RF power level. The fabrication cost of $\mathrm{SiGe}$ power HBTs can be thus be reduced by using a lithography of larger feature sizes and by using a reduced chip area. Therefore, such a base optimization approach can help make the SiGe BiCMOS technology more competitive than its current status in comparison to III-V counterparts for power amplifications.

Finally, while the principle of the base-region optimization has been demonstrated using mesa-type SiGe HBTs in this study, it can be applied to planar-type SiGe HBTs integrated on the SiGe BiCMOS technology.

\section{Conclusion}

With proper base-region optimizations (a higher base doping level than the emitter and a high Ge content of proper shapes) to realize a low base resistance for SiGe power HBTs, the microwave power amplification at high frequencies using these devices has been demonstrated to be feasible. The base-region optimizations can effectively improve the power-gain values of SiGe HBTs in a wide frequency range, maintain highbreakdown-voltage characteristics of SiGe power HBTs, enable the $\mathrm{CB}$ configuration to be more favorable for power amplifications with better ruggedness and further enhanced power-gain values, relax the lateral scaling of SiGe-power-HBT emitter stripes, and potentially reduce the cost of an SiGe BiCMOS for power amplification. As a result from such a base-region optimization, the highest power performance with an FOM of $7 \times 10^{4} \mathrm{~mW} \cdot \mathrm{GHz}^{2}$ was achieved from a single SiGe power HBT with emitter-finger stripes of $2-\mu \mathrm{m}$ wide.

\section{REFERENCES}

[1] J.-S. Rieh, B. Jagannathan, D. R. Greenberg, M. Meghelli, A. Rylyakov, F. Guarin, Z. Yang, D. Ahlgren, G. Freeman, P. Cottrell, and D. Harame, "SiGe heterojunction bipolar transistors and circuits toward terahertz communication applications," IEEE Trans. Microw. Theory Tech., vol. 52, no. 10 , pp. 2390-2408, Oct. 2004.

[2] M. Racanelli, P. Ma, and P. Kempf, "SiGe BiCMOS technology for highly integrated wireless transceivers," in Proc. Gallium Arsenide Integrated Circuits, San Diego, CA, Nov. 2003, pp. 183-186.

[3] J. Böck, H. Schäfer, K. Aufinger, R. Stengl, S. Boguth, R. Schreiter, M. Rest, H. Knapp, M. Wurzer, W. Perndl, T. Böttner, and T. F. Meister, "SiGe bipolar technology for automotive radar applications," in Proc. Bipolar/BiCMOS Circuits and Technology Meeting (BCTM), Montreal, Canada, Sep. 2004, pp. 84-87.
[4] N. Jiang, Z. Ma, G. Wang, P. Ma, and M. Racanelli, "3-W SiGe power HBTs for wireless applications," Elsevier Sci.: Mater. Sci. Semicond. Process., vol. 8, no. 1-3, pp. 323-326, 2005.

[5] M. Doherty, J. Harrison, M. McPartlin, and A. F. Quaglietta, "Power amplifier for CDMA cellular handsets delivers GaAs performance at silicon price," Microw. Product Dig. (MPD), p. 38, Sep. 2003.

[6] J. Pusl, S. Sridharan, P. Antognetti, D. Helms, A. Nigam, J. Griffiths, K. Louie, and M. Doherty, "SiGe power amplifier ICs with SWR protection for handset applications," Microw. J., vol. 44, no. 6, pp. 101-113, Jun. 2001.

[7] M. E. Kim, B. Bayraktaroglu, and A. Gupta, "HBT devices and circuit applications," in HEMTs and HBTs, Devices, Fabrication, and Circuits, F. Ali and A. Gupta, Eds. Norwood, MA: Artech House, 1991, pp. 301-302.

[8] E. O. Johnson, "Physical limitations on frequency and power parameters of transistors," RCA Rev., vol. 26, no. 2, pp. 163-177, Jun. 1965.

[9] N. Tanzi, J. Dykstra, and K. Hutchinson, "A 1-W doubly balanced $5 \mathrm{GHz}$ flip-chip SiGe power amplifier," in Proc. IEEE Radio Frequency Integrated Circuits (RFIC) Symp., Philadelphia, PA, Jun. 2003, pp. 141-144

[10] H.-F. Chau, D. Hill, R. Yarborough, and T. Kim, "High-power, highefficiency K-band AlGaAs/GaAs heterojunction bipolar transistors," in Proc. IEEE GaAs IC Symp., Orlando, FL, Nov. 1996, pp. 95-98.

[11] J. B. Johnson, A. J. Joseph, D. Sheridan, and R. M. Malladi, "SiGe BiCMOS technologies for power amplifier applications," in Proc. IEEE GaAs IC Symp., San Diego, CA, Nov. 2003, pp. 179-182.

[12] Z. Ma and N. Jiang, "On the operation configuration of SiGe HBTs based on power gain analysis," IEEE Trans. Electron Devices, vol. 52, no. 2, pp. 248-255, Feb. 2005.

[13] J. M. Rollett, "Stability and power-gain invariants of linear two ports," IRE Trans. Circuit Theory, vol. CT-9, no. 1, pp. 29-32, Mar. 1962.

[14] Z. Ma, S. Mohammadi, P. Bhattacharya, L. P. B. Katehi, S. A. Alterovitz, and G. E. Ponchak, "A high power and high gain $X$-band $\mathrm{Si} / \mathrm{SiGe} / \mathrm{Si}$ heterojunction bipolar transistor," IEEE Trans. Microw. Theory Tech., vol. 50, no. 4, pp. 1101-1108, Apr. 2002

[15] J.-S. Rieh, L.-H. Lu, L. P. B. Katehi, P. Bhattacharya, E. T. Croke, G. E. Ponchak, and S. A. Alterovitz, " $X$ - and Ku-band amplifiers based on Si/SiGe HBT's and micromachined lumped components," IEEE Trans. Microw. Theory Tech., vol. 46, no. 5, pp. 685-694, May 1998.

[16] A. Schüppen, U. Erben, A. Gruhle, H. Kibbel, H. Schumacher, and U. König, "Enhanced SiGe heterojunction bipolar transistors with 160 GHz- $f_{\max }, "$ in IEDM Tech. Dig., Washington, DC, Dec. 1995, pp. $743-746$.

[17] D. L. Harame, J. H. Comfort, J. D. Cressler, E. F. Crabbé, J. Y.-C. Sun, B. S. Meyerson, and T. Tice, "Si/SiGe epitaxial-base transistors-Part I: Materials, physics and circuits," IEEE Trans. Electron Devices, vol. 42, no. 3, pp. 455-468, Mar. 1995.

[18] K. D. Hobart, F. J. Kub, N. A. Papanicoloau, W. Kruppa, and P. E. Thompson, "Si/Si $\mathrm{Si}_{1-\mathrm{x}} \mathrm{Ge}_{\mathrm{x}}$ heterojunction bipolar transistors with high breakdown voltage," IEEE Electron Device Lett., vol. 16, no. 5, pp. 205-207, May 1995.

[19] [Online]. Available: http://public.itrs.net/Files/2001ITRS/SysDrivers.pdf

[20] A. Keerti and A. Pham, "SiGe power devices for 802.11a wireless LAN applications at $5 \mathrm{GHz}$," Electron. Lett., vol. 39, no. 16, pp. 1218-1220, Aug. 2003.

[21] L. D. Lanzerotti, J. C. Sturm, E. Stach, R. Hull, T. Buyuklimanli, and C. Magee, "Suppression of boron transient enhanced diffusion in SiGe heterojunction bipolar transistors by carbon incorporation," Appl. Phys. Lett., vol. 70, no. 23, pp. 3125-3127, Jun. 1997.

[22] I. M. Anteney, G. Lippert, P. Ashburn, H. J. Osten, B. Heinemann, G. J. Parker, and D. Knoll, "Characterization of the effectiveness of carbon incorporation in SiGe for the elimination of parasitic energy barriers in SiGe HBTs," IEEE Electron Device Lett., vol. 20, no. 3, pp. 116-118, Mar. 1999.

[23] J. Böck, H. Schäfer, K. Aufinger, R. Stengl, S. Boguth, R. Schreiter, M. Rest, H. Knapp, M. Wurzer, W. Perndl, T. Böttner, and T. F. Meister, "SiGe bipolar technology for automotive radar applications," in Proc. Bipolar/BiCMOS Circuits and Technology Meeting (BCTM), Montreal, Canada, Sep. 2004, pp. 84-87.

[24] T. Tominari, S. Wada, K. Tokunaga, K. Koyu, M. Kubo, T. Udo, M. Seto, K. Ohhata, H. Hosoe, Y. Kiyota, K. Washio, and T. Hashimoto, "Study on extremely thin base SiGe: C HBTs featuring sub 5-ps ECL gate delay," in Proc. Bipolar/BiCMOS Circuits and Technology Meeting (BCTM), Toulouse, France, Sep. 2003, pp. 107-110.

[25] O. Berger, "GaAs HBT for power applications," in Proc. Bipolar/ BiCMOS Circuits and Technology Meeting (BCTM), Montreal, Canada, Sep. 2004, pp. 52-55. 


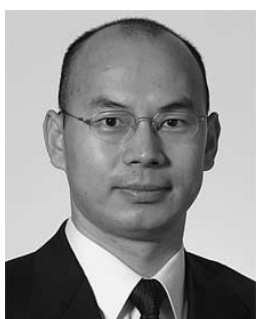

Zhenqiang Ma (S'98-M'01) received the B.S. degree in applied physics and the B.E. degree in electrical engineering from Tsinghua University, Beijing, China, in 1991, and the M.S. degree in nuclear science and the M.S.E. and Ph.D. degrees in electrical engineering from the University of Michigan, Ann Arbor, in 1997, and 2001, respectively. His doctoral research focused on the development of SiGe power heterojunction bipolar transistors (HBTs) for highfrequency microwave applications.

In June 2001, he joined the R\&D team of Conexant Systems and later its spin-off Jazz Semiconductor, Newport Beach, CA, where he worked on the development, characterization, and modeling of high-speed SiGe HBTs. In August 2002, he left Jazz to join the faculty of University of Wisconsin-Madison, as an Assistant Professor in the Department of Electrical and Computer Engineering. His research interests include SiGe and III-V HBT/FET devices and technologies, RF circuits, monolithic microwave integrated circuits, reliability of devices and circuits, $\mathrm{Si}$ and $\mathrm{SiGe}$ strained materials and physics, novel integration scheme, wide bandgap semiconductor materials, devices and applications, and nanoscale devices. He is the author of more than 50 peer-reviewed technical papers related to his research. He was featured in MIT's Technology Review magazine for his innovation on cell phone power devices in 2004. He served as a Technical Reviewer of Elsevier Superlattices and Microstructures, Semiconductor Science and Technology, Electrochemical and Solid-State Letters, Journal of Physics D: Applied Physics, IEE Electronics Letters, and Electronics and Telecommunications Research Institute Journal.

Dr. Ma served as a Technical Reviewer of IEEE TRANSACTIONS on Electron Devices, IEEE TRANSACTIONS ON Circuits AND SYSTEMS-II: EXPRESS BRIEFS, and IEEE TRANSACTIONS ON NANOTECHNOLOGY.

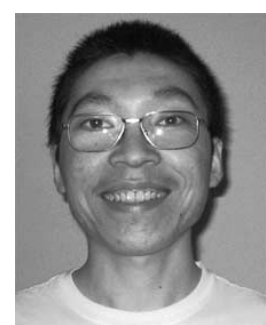

Ningyue Jiang (S'04) received the B.S. degree in electronic engineering from Tsinghua University, Beijing, China, in 2000 and the M.S. degree in electrical engineering from the University of New Mexico, Albuquerque, in 2002. He is currently working toward the $\mathrm{Ph} . \mathrm{D}$. degree in electrical engineering at the University of Wisconsin-Madison

His research interests include SiGe heterojunction-bipolar-transistor devices and circuits for $\mathrm{RF}$ and microwave applications. 